Ann. Abeille, I963, 6 (3), I67-183.

\title{
ESSAIS D'ENREGISTREMENT SIMULTANÉ DES PRINCIPAUX PHÉNOMÈNES DE LA VIE D'UNE RUCHE
}

\author{
R. CHAUVIN
}

Station de Recherches sur l'Abeille et les Insectes sociaux, Bures-sur-Yvette, (Seine-et-Oise)

\author{
SOMMAIRE
}

On décrit une ruche vitrée, avec un seul grand cadre, qui permet l'observation continue des abeilles, même pendant l'hivernage. Un contrôle thermique extérieur permet, à l'aide d'une résistance, d'agir à volonté sur le développement de la ruche.

Lne série d'appareils peuvent se brancher sur la même ruche, et enregistrent simultanément l'activité de sortie, la récolte de pollen, l'élimination d'eau. L'enregistrement de l'activité a permis notamment de découvrir qu'à certaines époques, se produisent des sorties d'abeilles pendant la nuit. On a mesuré en même temps la surface du couvain et ses fluctuations au cours de l'année.

Bien que les travaux sur l'ensemble de la ruche soient fort nombreux, on s'aperçoit avec étonnement qu'un grand nombre de variables sont bien mal connues, et surtout n'ont pas fait 1'objet d'enregistrements méthodiques et prolongés : par exemple l'activité des abeilles au cours du butinage : il n'existe (en comptant la mienne, déjà ancienne et incomplète) que deux ou trois publications sur le sujet ; le ramassage $d u$ pollen : beaucoup d'auteurs ont mesuré les quantités de pollen récoltées par jour, mais je ne crois pas qu'il existe un seul exemple d'enregistrement continu de l'apport de pollen (il est pourtant très facile, comme on le verra plus loin) ; la récolte $d u$ nectar: elle se mesure à l'aide de balances enregistreuses, mais les cas où on a pu la mettre en corrélation avec une mesure quelconque de l'activité, par exemple, sont bien rares. Or, ces variables sont tout à fait essentielles (sans parler d'autres non moins importantes, comme la température moyenne de la ruche); si l'on pouvait les exprimer en courbes, tout en observant à l'aise et sans aucune perturbation desi ouvrières à l'intérieur de la ruche, nul doute que l'apidologie ne fasse alors beaucoup de progrès.

Tel a été le but de mes travaux poursuivis pendant treize ans ; on verra plus loin qu'il était difficile à atteindre, mais non utopique. Je décrirai d'abord la ruche d'observation, puis les dispositifs d'enregistrement de l'activité, de la récolte du nectar et du pollen. L'ensemble de ces dispositifs se voit sur la planche photographique. 

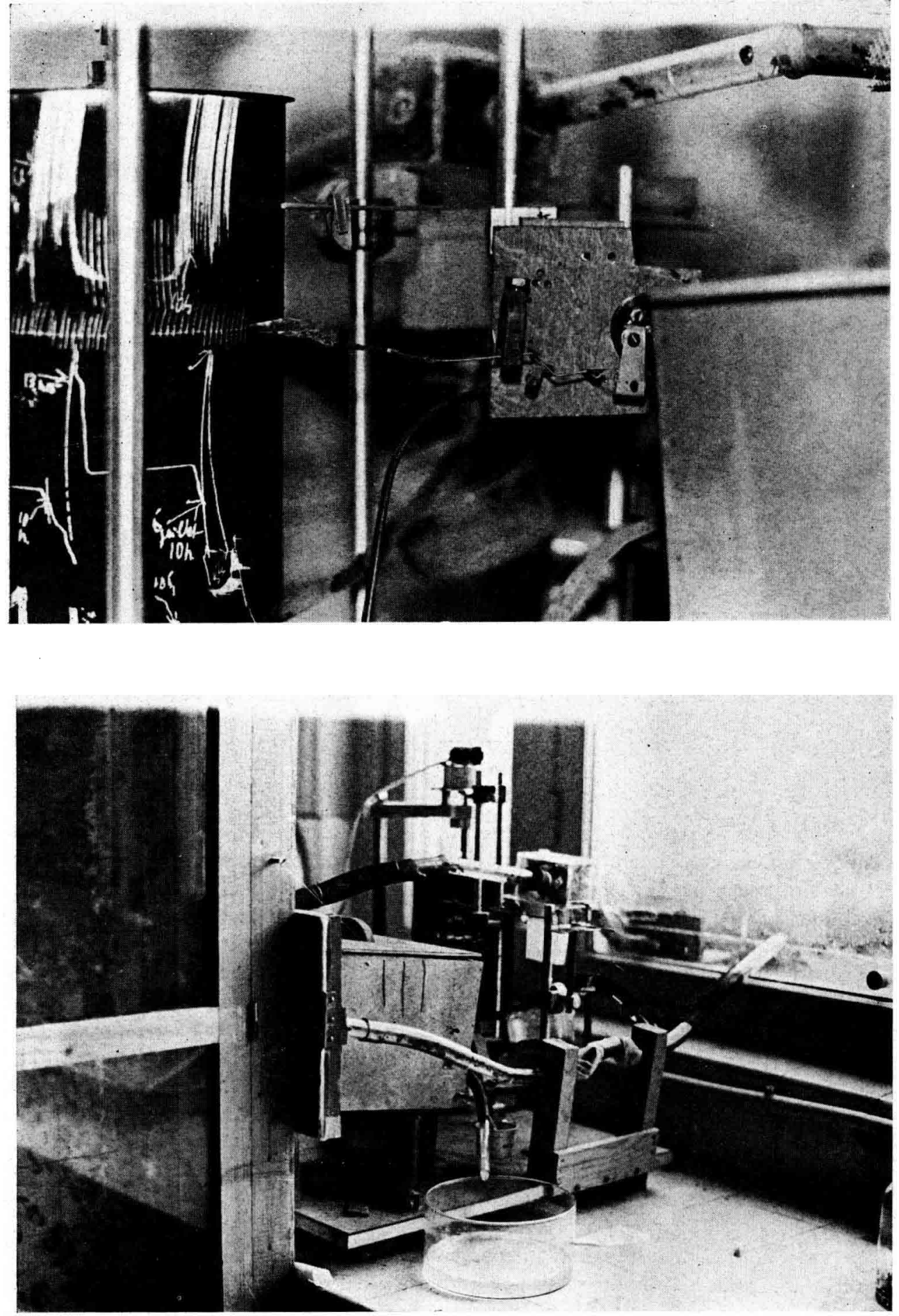

Daux vues de lensemble de lappareillage sous des angles différents 


\section{LA RUCHE D'OBSERVATION}

Les modèles de ruches d'observation sont très nombreux et il n'est guère de laboratoire d'apidologie qui n'en possède. Le modèle le plus connu est celui de von FrISCH. Il se compose de cadres de ruches placés les uns sur les autres, entre deux vitres qui peuvent être obturées par des panneaux de bois. Les abeilles sortent librement à l'extérieur. J'ai moi-même utilisé de tels modèles, mais mon but différait de celui des autres chercheurs, en ce que je voulais obtenir le maintien indéfini et en bonnes conditions de la colonie placée dans la ruche. J'ai constaté d'abord que de graves perturbations (arrêt de la ponte et diminution rapide de la population accompagnés souvent de désertion) se produisaient dans ces ruches dès que survenaient les jours froids. Ceci n'est pas étonnant et doit être attribué certainement à la formidable déperdition thermique dans ces conditions. Les abeilles montrent d'ailleurs un appétit dévorant et n'arrivent pas à emmagasiner assez de provisions, qui sont aussitôt consommées. Il était évident qu'il fallait un chauffage extérieur pour pallier ces inconvénients. Je le réalisai presque dès le début, en enfermant le dispositif, à l'intérieur d'une grande étuve chauffée à $35^{\circ}$. Quant aux rayons, je me rendis assez vite compte que la simple superposition de rayons empruntés à une ruche, les une au-dessus des autres, ne résolvait pas tous les problèmes. Pour des raisons que je ne suis pas arrivé à démêler, la reine refuse souvent de sortir du rayon où elle se trouve, et d'aller pondre sur les autres, et lorsqu'on l'en chasse, elle y revient. Si bien qu'un rayon est garni d'œufs jusqu'à la dernière cellule et les autres vides, et l'on n'y peut rien changer, même en introduisant des ponts de cire entre les rayons. La seule manière de procéder consiste alors à fixer les unes près des autres des feuilles de cire, de manière à constituer un seul et énorme rayon.

Il faut évidemment employer des fils métalliques plus épais que d'habitude pour armer la ruche qui est de grande dimension ; car les feuilles de cire insuffisamment maintenues par des fils trop minces sur une trop grande longueur pourraient s'affaisser. D'autre part, j'ai trouvé avantageux de constituer une ruche à une face, c'est-à-dire de rapprocher suffisamment la cire d'une des vitres pour que les abeilles ne puissent pas construire entre verre et cire. C'est d'ailleurs assez délicat car si les cires se trouvent trop près du verre, les abeilles les découpent et collent au verre les bords de l'ouverture ainsi pratiquée. L'espacement à observer est indiqué sur la figure 6 . Les dimensions de la ruche doivent être aussi respectées (au maximum $1 \mathrm{~m} \times 1 \mathrm{~m}$ ). Car j'en avais construit une plus grande de $2 \mathrm{~m} \times \mathrm{I} \mathrm{m}$ et bien que son développement ait été assez satisfaisant à la belle saison elle n'a jamais pu hiverner convenablement ni entreposer suffisamment de provisions. Ce qui tient sans doute, à des particularités du chauffage dans les ruches trop grandes. Nous avons vu en effet, que les abeilles dévoraient tout leur miel dans les ruches d'observation non chauffées.

\section{ACCESSOIRES}

La paroi transparente à travers laquelle se feront les observations est en matière plastique, de préférence en plexiglas, avec de place en place des orifices obturables par un couvercle à pivot. On peut ainsi atteindre les abeilles et les prélever le cas échéant. Il est bon de fixer sur le plexiglas, une caissette de $\mathrm{I} 5 \times \mathrm{I} 5 \times 5 \mathrm{~mm}$ où l'on 
peut introduire des abeilles pour renforcer la population ; le nourrisseur se compose d'un vase circulaire de $500 \mathrm{cc}$ avec flotteur de bois paraffiné. Les abeilles y ont accès par la partie supérieure et sucent le sirop sans s'y noyer.

\section{PEUPLEMENT ET CONDUITE DE LA RUCHE EXPÉRIMENTAI,E}

Il s'opère au premier printemps par l'introduction d'un kilo et demi d'abeilles anesthésiées au gaz carbonique, dans la caisse d'introduction : la reine est posée sur le dessus pour éviter l'écrassement sous la masse des ouvrières endormies. On monte aussitôt la température à $+34^{\circ}$ et on nourrit abondamment au sirop tiède. L' 'étirage de la cire gaufrée a lieu presque aussitôt et la ponte doit survenir dans les huit jours.

La surveillance de la ponte est ici moins facile que dans la ruche transparente que j'avais décrite en I950. Dans cette dernière, à l'aide d'une technique assez simple, les feuilles de cire gaufrée étaient collées sur une plaque de verre. Alors les ouvrières enlèvent presque complètement le fond de cire de chaque cellule et on voit très facilement par transparence la reine pondre; rien n'est plus aisé que de compter les cufs jour par jour. Mais il n'est pas facile de réaliser un tel dispositif avec de grandes surfaces et j'ai préféré celui qui se trouve décrit plus haut. De toute façon, la surface d'observation est divisée en carreaux équidistants à l'aide desquels il est très aisé de repérer la variation de la surface de ponte et surtout de la surface occupée par le couvain operculé. La température doit être maintenue à $34^{\circ}$ pendant toute la période d'activité des abeilles, soit, dans le climat parisien, entre avril et septembre. Fin septembre, on baisse très progressivement de $4^{\circ}-5^{\circ}$ par semaine jusqu'à + I $8^{\circ}$, qui doit être atteint en octobre. On assiste alors à la formation de la grappe d'hiver. Il convient pendant tout l'hiver de ne pas laisser tomber la température en dessous de $I 8^{\circ}$; et si le besoin s'en fait sentir, de ne nourrir qu'au sucre candi et non pas au sirop qui cause des sorties intempestives même par temps froid. A partir de la reprise de l'activité de ponte (en fin février sous le climat parisien), il faut augmenter à nouveau la température de $I^{\circ}$ à $2^{\circ}$ par semaine, un peu plus si le temps est chaud et permet les sorties. Il ne faut pas atteindre $34^{\circ}$ avant le milieu ou même la fin avril.

\section{IA MESURE DE I'ACTIVITÉ}

La mesure d'une variable aussi importante que l'activité, a fait l'objet de deux tentatives principales; celle de LUNDIE et celle de FABERGE. Les deux auteurs ont employé la même technique : les abeilles poussent de petits leviers qui déclenchent un contact électrique. On compte ensuite, soit manuellement soit automatiquement, le nombre des contacts. Mais cette technique qui paraît simple, est en réalité d'une application très difficile. Les efforts que l'abeille exerce sur le levier, sont forcément très faibles, d'où la nécessité d'un mécanisme. très délicat qui est exposé pourtant à l'air et surtout à l'abeille : c'est bien là le plus grave, car tôt ou tard, les ouvrières déposent sur tous les corps avec lesquels elles se trouvent en contact, des épagines (ChauviN, I960) dérivées de la propolis ou d'autres substances, qui encrassent irrémédiablement tous les mécanismes. D'autre part, on est forcé d'alimenter les contacts en bas voltage, et il faut savoir qu'il peut sortir d'une ruche de 
I 5 à 20 ooo butineuses par jour. A moins que la construction ne soit parfaite, les multiples petites étincelles qui jaillissent à l'occasion des mouvements des leviers, finissent par abîmer les surfaces de contact qu'il faut nettoyer très souvent. LundiE écrit lui-même que le plus clair de son temps était occupé à tenir le dispositif en ordre de marche. Si bien qu'il n'est guère utilisable dans la pratique.

J'ai essayé moi-même plusieurs méthodes d'enregistrement de l'activité des abeilles et certains de mes échecs sont instructifs, c'est pourquoi je les décrirai brièvement; ce n'est qu'en dernier lieu (Ig62) que j'ai pu mettre au point un dispositif extrêmement simple, satisfaisant les besoins de l'expérimentateur.

Io) J'ai décrit d'abord, en I952, un dispositif hydraulique qui me paraissait assez convenable. L'abeille devait sortir de la ruche à travers un tube de verre en

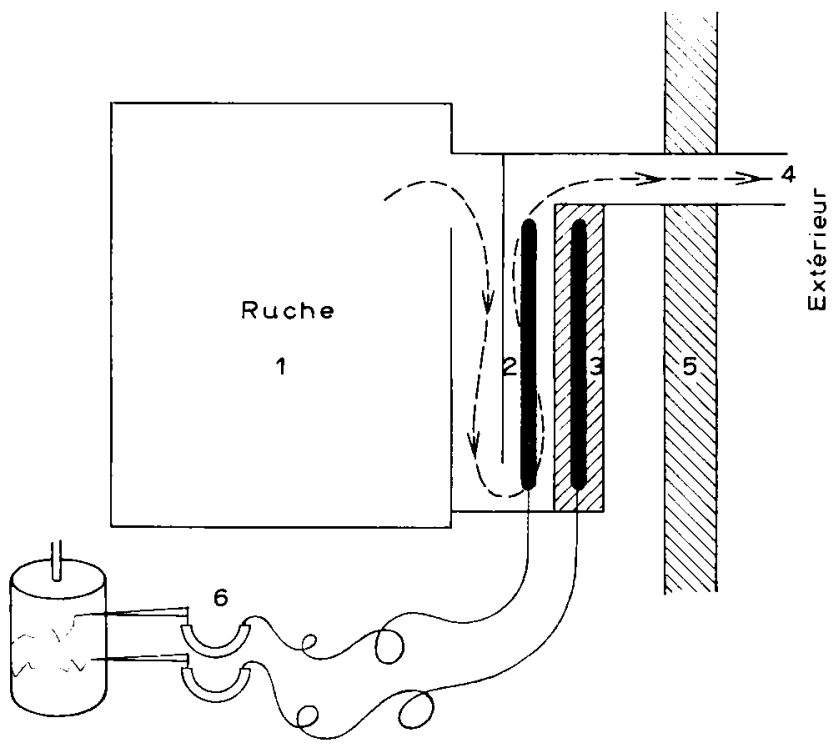

FIG. I. - .- Monlage des enregistreurs thermiques dans un dispositif a chicanes, ne laissam passer les abeilles quautour d whe seule des deux sondes

I, ruche. 2, sonde autour de laquelle jassent les abeilles. 3 , sonde témoin. 4 , trajet des abeilles figuré par une ligne pointillée avec flèches. 5 , mur du liboratoire. 6 , dispositif enregistreur

poussant un petit levier. Le levier soulevait légèrement une boule de métal qui ouvrait un petit orifice au fond d'un récipient d'eau, à niveau constant. 'Toutes les gouttelettes tombaient dans un vase muni d'un flotteur. Un style fixé au flotteur, inscrivait ses déplacements sur un cylindre tournant. Enfin, un siphonage périodique évacuait l'eau autour du flotteur, lorsque ce dernier se trouvait au sommet de sa course. L'appareil ainsi conçu a fonctionné plusieurs années, mais ce n'est qu'après l'avoir beaucoup perfectionné que je me suis aperçu d'une cause d'erreur d'autant plus grave qu'elle tenait au principe même de 1'appareil : les abeilles ne poussent pas toujours le levier de la même façon, et ceci tient aux variations de la température extérieure. Lorsqu'il fait chaud, elles poussent brusquement le levier et passent rapidement, et ceci ne correspond alors qu'à l'émission d'une petite quantité d'eau. Mais, lorsque 
la température fraîchit, les abeilles passent très lentement, ne poussent le levier qu'après bien des hésitations, et on n'a plus aucun moyen de savoir à combien de passages correspond la même quantité d'eau écoulée à des jours différents. Je n'ai pu trouver le moyen de corriger cette cause d'erreurs, et $j$ 'ai $d \hat{\imath}$, par conséquent, abandonner un dispositif qui m'avait inspiré, pour un temps, beaucoup d'espoir.

$2^{\circ}$ ) Les abeilles, qui rentrent à la ruche, viennent de voler et, par conséquent, leur musculature thoracique s'est fortement échauffée. On sait qu'on a pu mesurer, après le vol, chez les ouvrières et les mâles, des températures qui dépassaient parfois $40^{\circ}$. Il s'ensuit que le trou de vol est traversé sans arrêt par un certain nombre de machines calorifiques, et que, si l'on pouvait recueillir la chaleur dégagée, on y trouverait un bon indice de l'activité de la ruche. Je dirai, tout d'abord, que cet apport de chaleur est effectivement considérable puisque le dispositif le plus grossier suffit à le mettre en évidence. Si, par exemple, dans le dispositif de la figure I et à la place des sondes thermiques, on intercale deux tubes gradués verticaux et pleins d'éther, dont l'un est protégé des abeilles alors qu'elles peuvent passer autour de l'autre, on s'apercevra très vite que le niveau de l'évaporomètre placé du côté des abeilles, baisse bien plus vite que celui de l'autre. Le tableau ci-contre :

Niveaux de l'éther dans les évaporomètres (en centicubes)

\begin{tabular}{|c|c|c|c|}
\hline $1^{\text {er }}$ tube & d)assage des abeilles. & Tube témoin & Diff. \\
\hline 17 sept. $1959, \ldots \ldots \ldots$ & 7.7 & 9,3 & 1,6 \\
\hline 18 sept. $1959 \ldots \ldots \ldots$ & 4,5 & 5,9 & $1, t^{\prime}$ \\
\hline 19 sept. $1959 . \ldots \ldots \ldots$ & 1,9 & 2,8 & 0,8 \\
\hline$\geq_{0}$ sept. $1959 . \ldots \ldots \ldots$ & 5,8 (remplissage à 10 le 19 ) & 6,8 & 1 \\
\hline 21 sept. $1959 . \ldots \ldots \ldots$ & 2,5 & 3,5 & 1 \\
\hline 2. sept. $1959 \ldots \ldots \ldots$ & 7,3 (remplissage le 21 ) & 8,4 & 1,1 \\
\hline 93 sept. $1959 \ldots \ldots \ldots \ldots$ & $: 3,\}^{\prime}$ & $\{, 9$ & \\
\hline
\end{tabular}

correspond à 5 jours qui ont débuté le I7 sept. I959. On peut aussi placer (fig I) deux grandes sondes thermiques (tubes creux, pleins de tétrachlorure de carbone), l'un

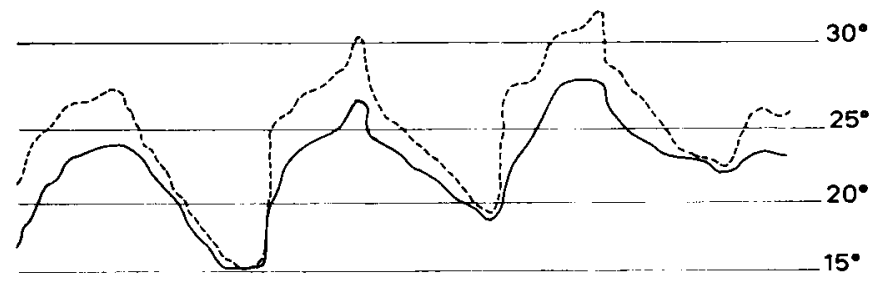

FIG. 2. - Tracé des enregistreurs thermiques. La ligne pointillée correspond a la sonde 2 , la ligne en traits pleins à la sonde 3. Trois jours successits (juillet $\mathrm{r}_{960}$ )

au milieu des abeilles qui entrent et sortent, et l'autre comme témoin. Là aussi, une très forte et très constante différence de température correspond aux périodes d'activité (fig. 2). Toutefois, ces deux dispositifs m'ont paru impropres à des mesures précises. Les chiffres qu'ils fournissent ne sont pas en corrélation tant s'en faut, 
avec ceux que l'on trouve par l'observation directe, ou bien ils n'y sont qu'assez rarement; l'évaporation en effet, est un phénomène délicat difficile à interpréter et les différences ne sont pas de la même valeur suivant la bande des températures à laquelle on se place. De plus, il est probable que l'échauffement du thorax de l'abeille n'est pas non plus le même suivant la température de l'air où elle se déplace.

$\left.3^{\circ}\right)$ Un dernier dispositif, et celui qui donna les résultats les plus satisfaisants,

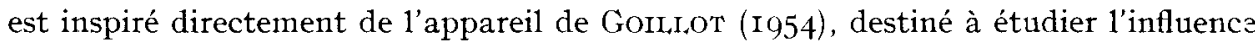
de la fumée sur les ouvrières. Il se compose (fig. 3) d'un cylindre très léger, horizontal, suspendu à un style qui l'équilibre. Le cylindre peut se déplacer sans frottement dans une caissette noircie. Les abeilies, pour sortir, sont à peu près toutes forcées de passer à l'intérieur du cylindre ou de marcher sur sa face extérieure. Dans les deux

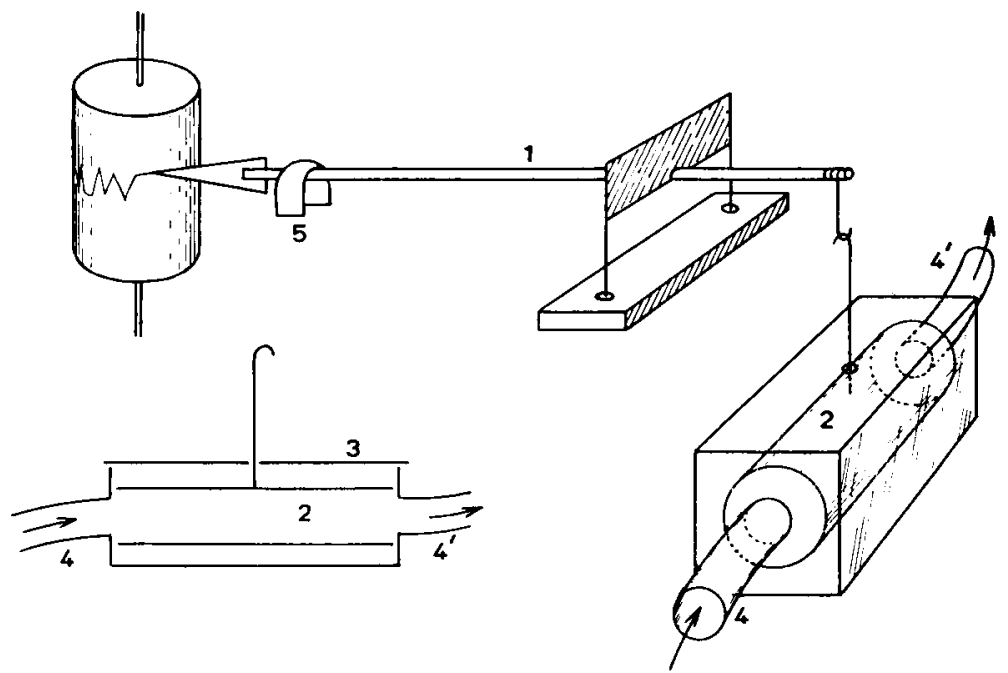

FIG. 3. . Enregistrement de l'acizité de sortie

I, levier avec contrepoids (5). 2, cyclindre de métal léger enfermé dans la boîte 3 , et à travers lequel passent les abeilles en 4 et $4^{\prime}$. Les extrémités du cylindre doivent être à 3 millimètres des parois de 3 .

cas, le résultat cherché est atteint, c'est-à-dire que le cylindre est déséquilibré et inscrit un trait sur un cylindre enregistreur enfumé ; la hauteur de ce trait est grossièrement proportionnelle au nombre d'abeilles qui se trouvent dans ou sur le cylindre au même moment (la hauteur maximum correspond à 7 abeilles passant en même temps). On admet que la proportion des abeilles qui sont détectées par le cylindre est une fraction à peu près constante de l'ensemble de la population en transit, dont une partie marche sur les parois de la boîte extérieure et n'agit donc pas sur l'inscripteur. Cette conclusion est corroborée par des comptages qui montrent que l'erreur que l'on peut commettre ainsi, n'est pas considérable, bien qu'il ne soit pas aisé de 1'évaluer avec exactitude. Mais j'ai fait remarquer (I952), que lorsque Io ooo abeilles vont butiner, il n'importe pas beaucoup que l'on en compte I I ooo ou 9 ooo; car nos méthodes, hélas! n'en sont qu'au début et ne peuvent encore s'offrir le luxe d'une grande précision. 


\section{L'ENREGISTREMENT DES RÉCOLTES DE POLLEN}

Il n'offre par contre aucune difficulté. Il suffit, conformément à la figure 4, de placer en dessous d'un petit piège à pollen d'un modèle très classique (voir CHAUviN, I955), un récipient fixé au fléau d'une petite balance enregistreuse, et on obtient sans difficulté, l'enregistrement des récoltes en fonction du temps.

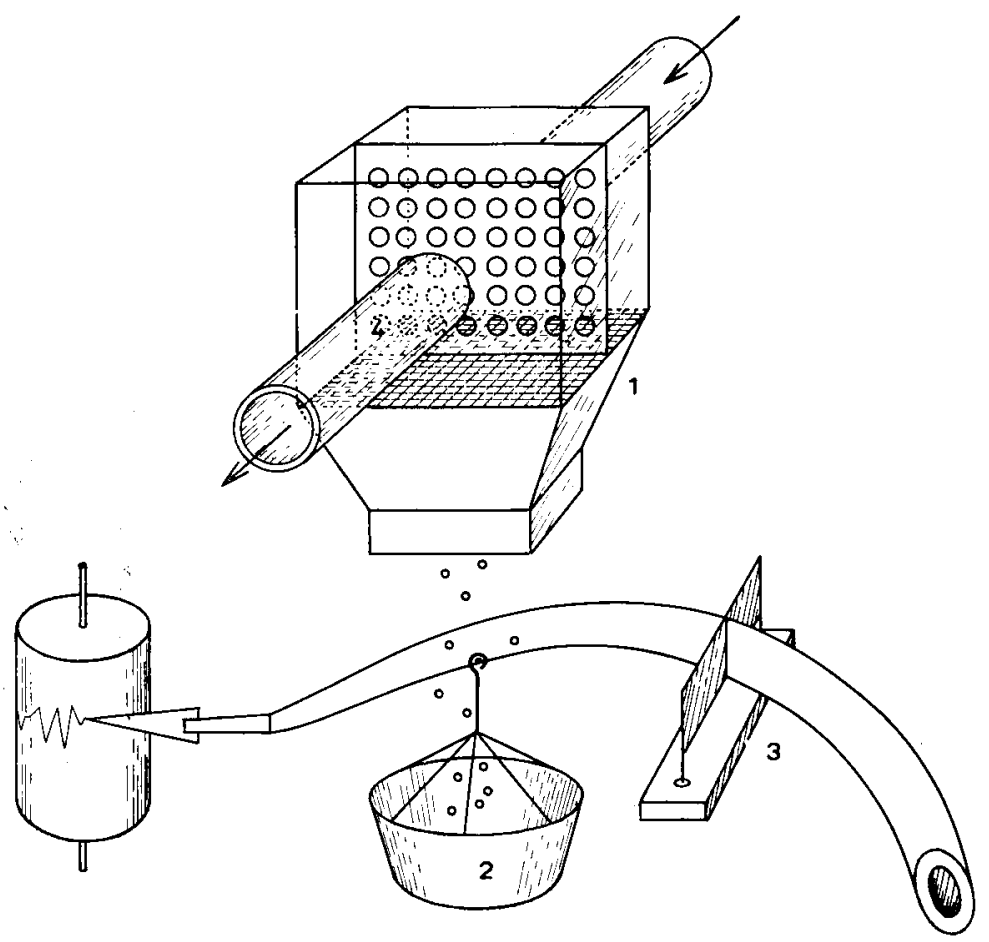

FiG. 4. - Enregistremenl de la récolte du pollen. Les abeilles rentrant à la ruche passent à travers le piège a pollen (I). Elles abandonnent leurs pelotes de pollen en passant à travers les trous de la plaque médiane; ces pelotes lombent à travers une grille en 2. En 3, disposition de la balance enregistreuse.

\section{L'ENREGISTREMENT DE LA RÉCOLTE DE; NECTAR}

Celui-ci est beaucoup plus difficile; il s'opère classiquement sur les ruches normales avec une bascule enregistreuse. Il est beaucoup plus délicat ici, où la taille et l'encombrement considérable des dipositifs d'enregistrement, interdisent pratiquement l'emploi d'une bascule. J'ai donc pensé procéder indirectement en récoltant une partie de l'eau évaporée par la ruche, et dont la quantité augmente forcément en temps de miellée. L,e nectar, en effet, doit être très fortement concentré avant de se transformer en miel operculé. Je ne puis encore garantir le dispositif que je vais décrire, ne l'ayant pas expérimenté assez longtemps; mais il donne des résultats encourageants. Il s'agit, en bref, de présenter à l'entrée de la ruche (fig. 6) un large tube métallique, fermé par une toile métallique pour en empêcher l'accès aux abeilles. I1 plonge dans une caisse à glace, et 1'eau qui s'y condense est recueillie dans une 
petite balance enregistreuse (fig. 5). Tout ce que je puis dire, c'est qu'effectivement, comme on peut le voir sur les graphiques, la quantité d'eau recueillie varie dans de très larges proportions et qu'elle augmente effectivement beaucoup, lorsque survient la miellée. En dehors de cette période, on peut, en administrant par exemple, un litre de sirop, calculer à quoi correspond l'eau que l'on recueille dans la balance enregistreuse. On verra un peu plus loin, une discussion plus approfondie de ces données.
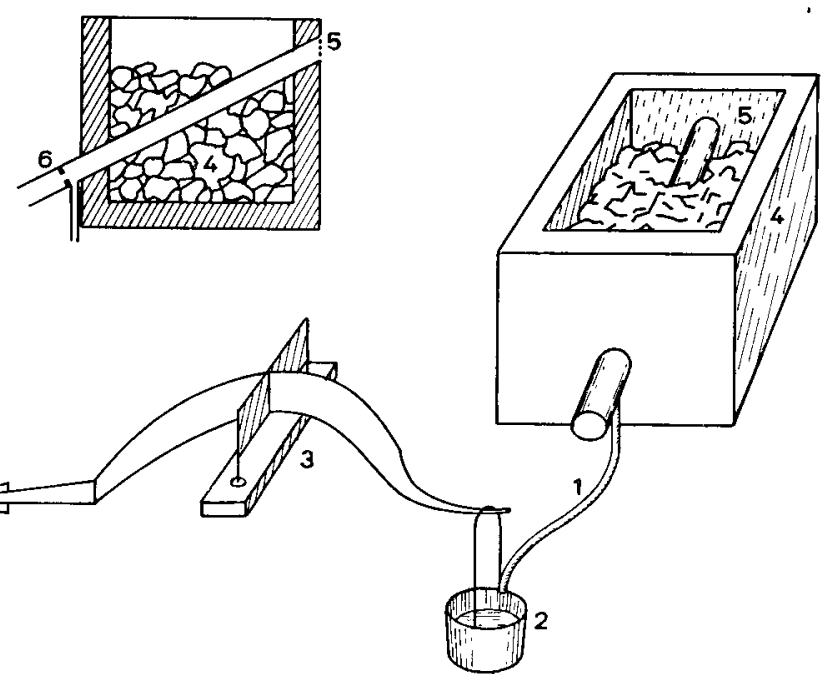

FIG. 5. - Enregistrement de l'élimination de vapeur d'eau de la ruche. L'air de la ruche passe d iravers une grille (5) dans un tube de cuivre oblique, immergé dans une bốle ḋ glace 4. L'eau de condensation est arrêtée en 6 par une saillie interne et passe par le tube I dans le récipient 2 . En 3, disposition de la balance enregistreuse.

\section{ANNEXE : LE DISPOSITIF DES FOLOPPE}

Comme on le verra sur les dessins, les abeilles ont à suivre, à travers tous ces dispositifs d'enregistrement, un chemin compliqué (fig. 7). L'activité a été mesurée uniquement sur les abeilles en train de sortir, et la récolte du pollen, évidemment, sur celles qui rentrent. Il a donc fallu séparer en deux le courant des abeilles, ce qui est facile à l'aide d'une variante du dispositif des frères FoLopPE, bien connue en apiculture. La sortie et l'entrée se font à travers deux gros tubes de verres, mais le tube de sortie qui s'ouvre au ras de la paroi de la ruche, en face des abeilles, fait largement saillie à l'extérieur vers l'air libre. Le tube de rentrée, au contraire, s'ouvre au ras du mur extérieur, mais après avoir traversé la paroi de la ruche, s'incurve longuement vers le bas. Lorsque les abeilles sont de retour du butinage, elles s'abattent au ras du mur extérieur: mais le tube de verre qui en sort n'a pas, pour elles, de signification, car le verre est transparent. Elles cherchent donc près de sa base et, trouvent ainsi 1'orifice de rentrée situé à proximité immédiate ; elles 1'utilisent aussitôt. L'incurvation du tube de rentrée à l'intérieur de la ruche les empêche de l'utiliser pour la sortie (fig. 7). 


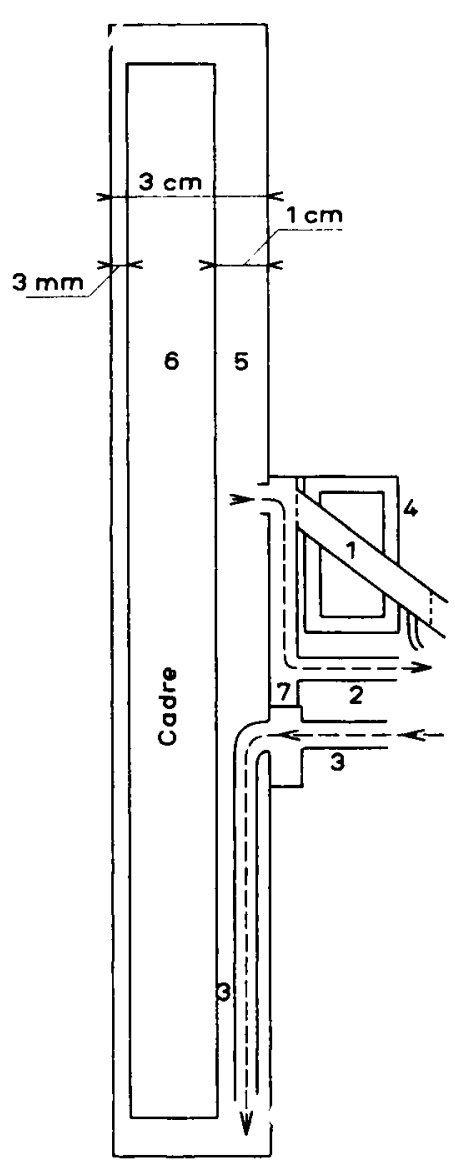

Fig. 6. - Coupe verticale de la ruche el du dispositif qui condense l'eau éliminée. On voit en $\mathrm{I}$, le tube de cuivre oblique dans sa caisse à glace 4. En 2 , une fieche pointillée montre le chemin que doivent suivre les abeilles pour sortir a travers le tube 7 . En 3 , chemin de la rentrée. 5 , ruche ; 6 , cadre de la ruche qui supporte la grande feuille de cire.

\section{RÉSULTATS}

\section{LE DÉVELOPPEMENT DE LA RUCHE EXPÉRIMENTALE AU COURS DE L'ANNÉE}

Je l'ai estimé en mesurant la surface du couvain operculé. Par suite de toute une série de contretemps, la courbe obtenue en $196 \mathrm{I}$ comprend plusieurs incidents. L'un, aux environs du 5 juin, correspond à l'emballement de la reine que je n'ai pas pu sauver à temps. Le second, le 20 juillet, est un arrêt de ponte brutal dû à une expérience aux conséquences inattendues : $j$ 'avais distribué aux abeilles, une macération de vieux rayons très noirs et contenant un peu de pollen; sa teneur en sucres avait été amenée à $70 \mathrm{p}$. Ioo. Je constatai avec beaucoup d'étonnement que les 
abeilles, très avides de sirop pourtant, ne prenaient celui-là qu'avec beaucoup de répugnance, et plus du tout au bout de 4 à 5 jours. La récolte du pollen s'interrompait radicalement, et les abeilles se massaient vers la partie supérieure du rayon. J'ai constaté par la suite, sur une ruche Dadant du type ordinaire, que les vieux rayons noirs et mélangés à du miel, ne sont pas acceptés non plus. Sans doute faut-il y voir l'action $\mathrm{du}$ substances répulsives dont l'origine et la nature chimique sont ma1 connues et dont l'accumulation provoque l'encrassement des vieux rayons. Il est curieux de noter qu'ils provoquent l'arrêt de la ponte de la reine (voir CHAuvin, Ig62) (fig. 8).

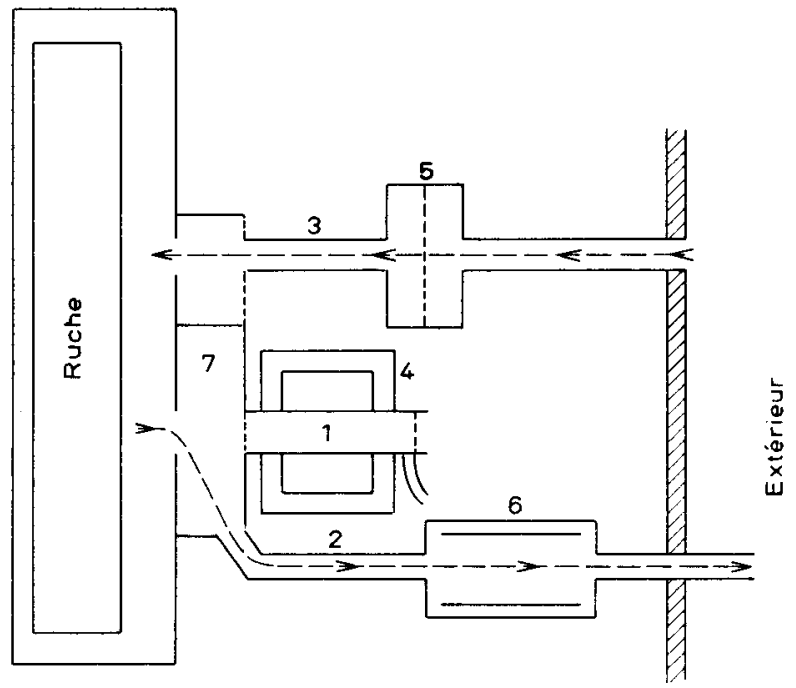

Fic: 7. - Coupe horizontale des memes appareils, avec détail du chemin suini par les abeilles. En 3, rentrée: les abeilles djivenl passer à travers le piège à pollen 5 . En 2 , sortie : les abeilles passent à travers la boîte 7 dezant le tube de cuive $\mathrm{I}$ dans sa caisse a glace 4 . Elles tranchissent ensuite en 6 , le cylindre qui sert d̀ mesurer leur activité.

En I962, la courbe de développement de la ruche est beaucoup plus régulière mais elle commence à monter très tard, vers la mi-mai à cause du très mauvais temps et de l'absence de toute miellée. Elle s'abaisse brutalement au début de septembre. Il n'y a que peu à dire sur ces courbes, assez différentes d'une année à l'autre suivant les conditions météorologiques. Il serait évidemment intéressant de les mettre en corrélation précise avec l'activité et l'élimination de vapeur d'eau ; malheureusement mes dispositifs enregistreurs se sont trouvés au point trop tard, et je dispose encore de trop peu de chiffres pour établir de telles corrélations (fig. 8).

Toutefois, je dois signaler que dans la ruche de trop grandes dimensions dont je me suis servi, le développement du couvain ne m'a pas paru satisfaisant. Les zones centrales du grand rayon de cire sont mal étirées ou pas du tout, et la reine parait se confiner dans ses portions supérieures et latérales. Dans les ruches de plus petites dimensions (surface approximative : I mètre carré) le développement est bien plus satisfaisants ; 1'ensemble de la cire peut être étiré en une seule nuit, et il n'est pas rare d'obtenir un énorme rayon de couvain d'à peu près I mètre de superficie. Je ne sais à quoi attribuer les anomalies observées dans la grande ruche ? Peut-êtreà 

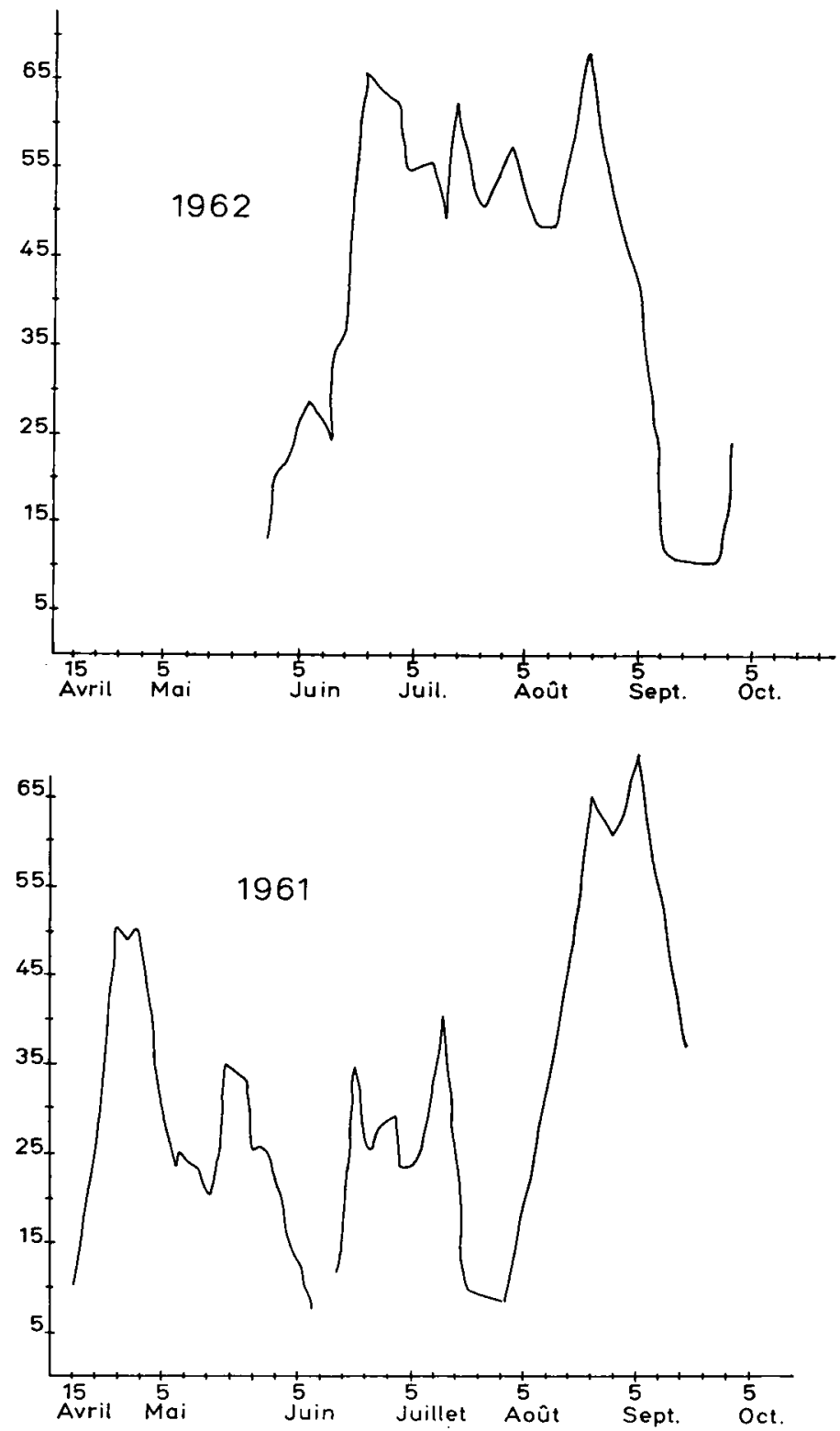

FIG. 8. - Surface de couvain operculé, au cours de deux années d'expérience dans lut ruche expérimentale en ordonnées, surface de couvain planimitrés.

une répartition anormale de la température? En tout cas, les surfaces maxima planimétrées dans cette ruche, correspondaient à $\Upsilon 200 \mathrm{~cm}^{2}$, alors qu'une belle ellipse de couvain (double face) sur un seul cadre Dadant occupe cette même surface ; sur la ruche de I mètre carré, on obtenait des ellipsse de 6 à 7 ooo centimètres carrés. 


\section{L'ACTIVIT'É}

Cette variable très importante n'a pratiquement été étudiée, avec quelques détails, que par un seul auteur, LUNDIE (I925). Plus tard, FABERGE apportera quelques perfectionnements à l'appareil de Luxdie, mais ne paraît pas l'avoir réellement mis en œuvre pour de nouvelles mesures. J'ai tenté moi-même quelques mesures mais je considère que, malgré dix ans d'efforts, 1'appareil que j'ai imaginé, n'a fonctionné d'une manière régulière que pendant quelques mois seulement. De plus, il ne fournit pas exactement une mesure d'activité directe, mais plutôt une grandeur qui varie suivant une loi encore inconnue en rapport avec l'activité. Je suis donc encore incapable d'établir, en nombre d'abeilles, à quoi correspondent mes graphiques. Je sais seulement, que pour la petite balance, telle qu'elle est constituée (grand bras du levier : $25 \mathrm{~cm}$ en paille sèche très légère ; petit bras : $8 \mathrm{~cm}$; contre poids à l'extrémité du grand bras $1,6 \mathrm{~g}$; poids du cylindre à travers lequel passent les abeilles : 5 grammes), il faut que passent 7 abeilles au moins, soit $700 \mathrm{mg}$ sensiblement, pour déséquilibrer la balance et amener le style au plus haut de sa course. On peut obtenir, évidemment, la sensibilité que l'on veut en changeant le contrepoids. On s'aperçoit qu'il faut le faire lorsque le style inscripteur reste longtemps sans redescendre, ce qui signifie qu'il passe en permanence plus de 7 abeilles. En général, et pour les conditions de mes expériences en I96I-62, il m'a semblé qu'un contrepoids de $\mathrm{r}, 6 \mathrm{~g}$ était suffisant. Mais le phénomène le plus intéressant qui nous est apparu d'une manière tout à fait inattendue, c'est l'activité nocturne (fig. 9, $a, b$ ). On en voit déjà quelque peu dans la nuit du I 4 au I 5 sept. I 962 , mais fort peu d'abeilles sont engagées dans 1'appareil, qui n'enregistre que de faibles différences de niveau. Par contre, dans les nuits du 26 au 27 , du 27 au 28 , et surtout du 28 au 29 , on constate de sérieuses poussées d'activité nocturne, à différentes heures de la nuit. Ces poussées ont été enregistrées aussi en I96I (fig. Io) (nuit du 5 au 6 juillet, et du 6 au 7 , par exemple, avec un autre appareil). Si on peut admettre qu'elles correspondent à une intense excitation des abeilles, du fait de la miellée en I.96r, on ne peut avancer une telle explication pour le mois de septembre I962. Nous ne savons comment interpréter le phénomène qui ne se produit qu'au cours des nuits chaudes; de plus, le I 4 sept. I 962 était jour de pleine lune. Mais nous n'avons pu retrouver de corrélation bien nette avec la lune et les sorties nocturnes en I96I; d'autre part, les tracés de I96I, n'étaient pas assez bons ni surtout assez réguliers pour autoriser des conclusions bien fermes. Le problème de l'activité nocturne des abeilles reste donc posé, quant à son déterminisme au moins.

Nous ne connaissons guère mieux les facteurs de l'activité diurne. Dans la fig. II, on a porté en ordonnées les "surfaces d'activité " intégrées : c'est-à-dire, que la surface occupée par les courbes d'activité a été calquée sur papier transparent, découpée et pesée. Au-dessous, on voit les valeurs correspondantes pour les températures intégrées (thermomètre enregistreur de RICHARD; en prenant comme base la ligne $0^{\circ}$, on décalque sur papier transparent et on pèse la "surface thermique " correspondant à chaque jour). On voit bien que les températures et les activités n'ont pas de rapport. Les chiffres donnés jour par jour par l'actinomètre de BELLANI (fig. I I) ne nous montrent non plus aucune corrélation avec l'activité. Les conditions de mon expérience étaient toutefois si spéciales que je me suis demandé si je ne me 

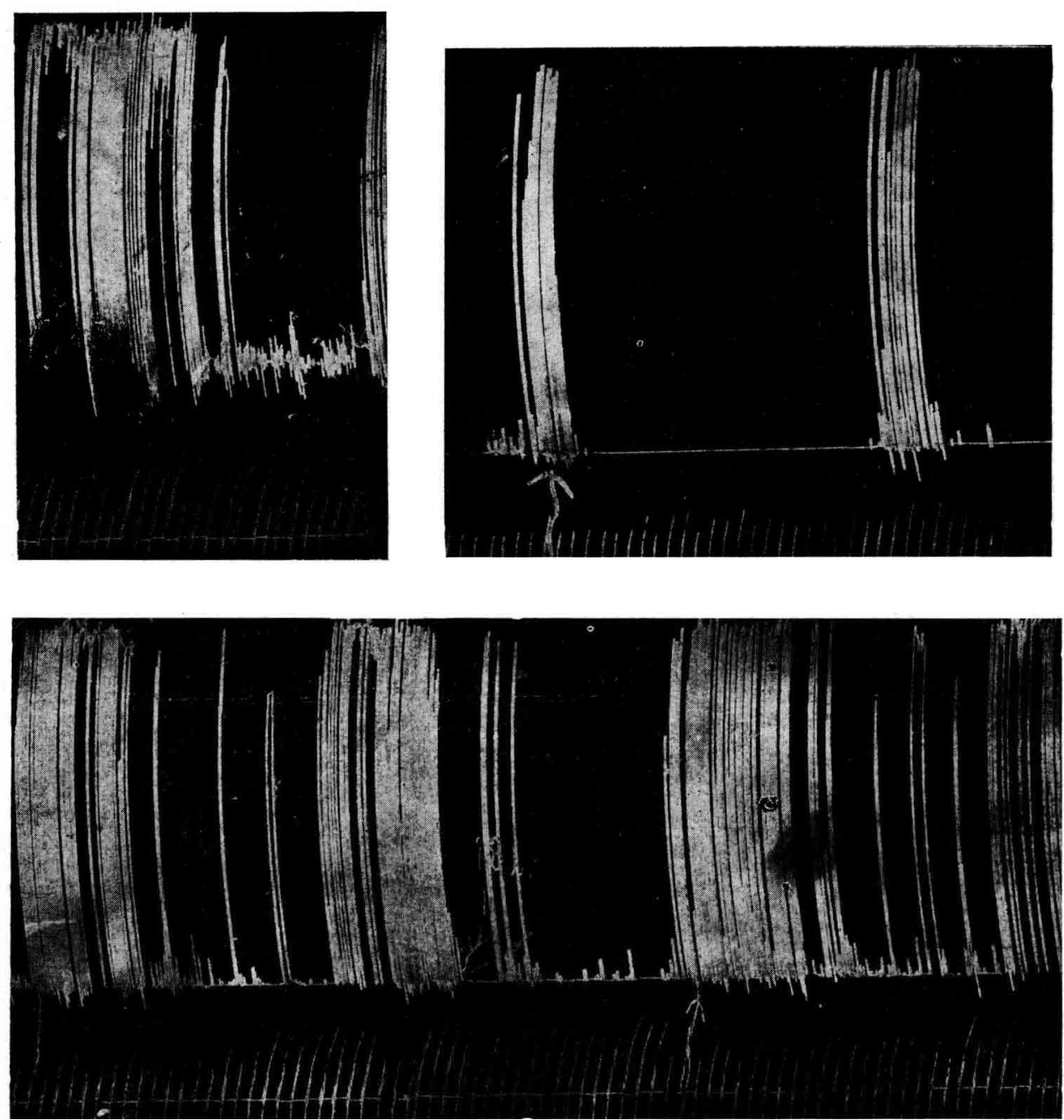

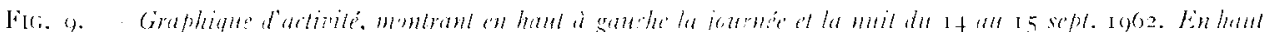

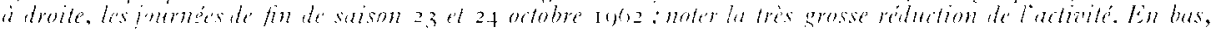

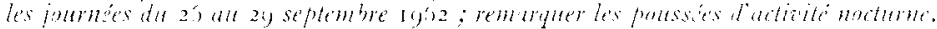

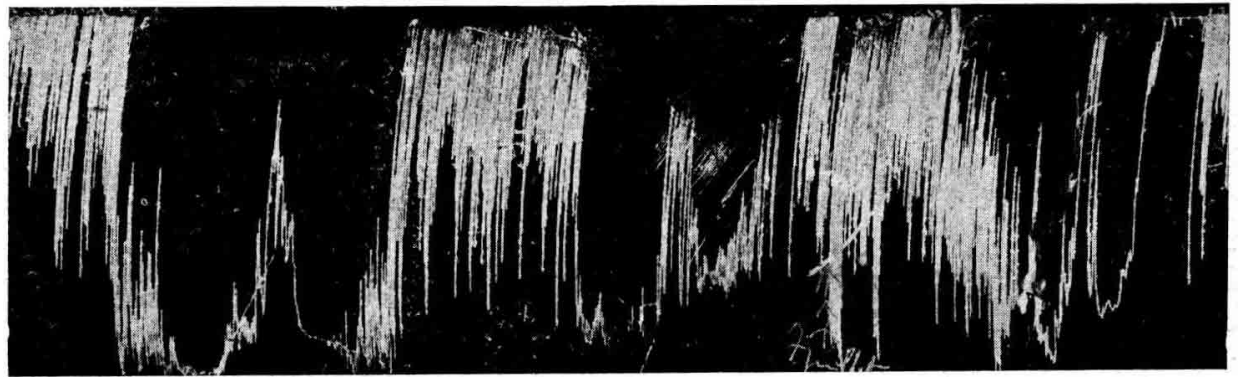

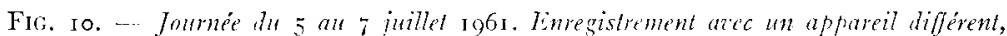
qui montre également l'artizité noclurne. 


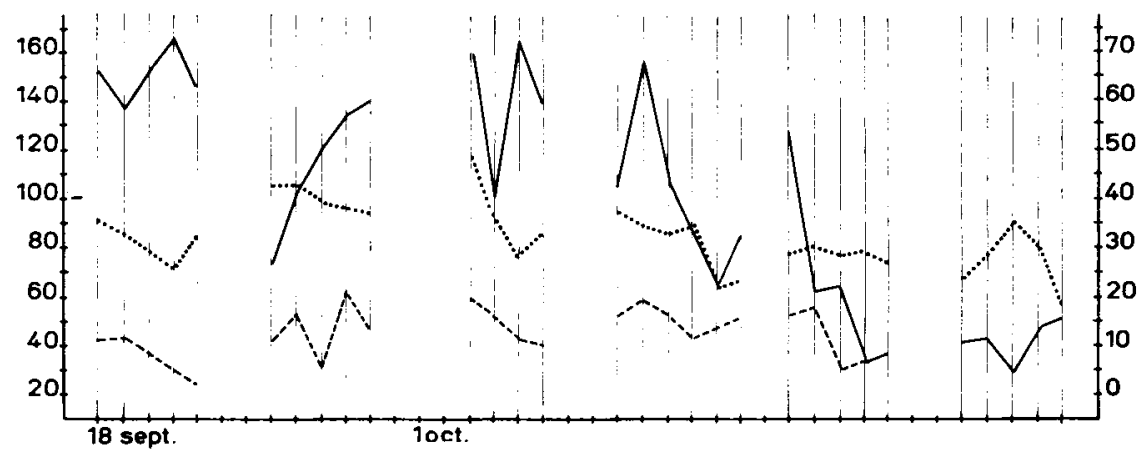

FIG. It. - Essais de corrélation entre l'activité des abeilles, la température et l'activité solaire. En traits pleins activité globale des abeilles (pour chaque jour on mesure la surface occupée par l'ensemble de la courbe d'enregistrement de l'activité). En pointillé, la température (on mesure la surface occupée par la courbe thermique de l'enregistreur Richard, en prenant comme origine la ligne de $\left.0^{\circ}\right)$. En tirelé radiations totales mesurées a l'actinomètre Bellani.

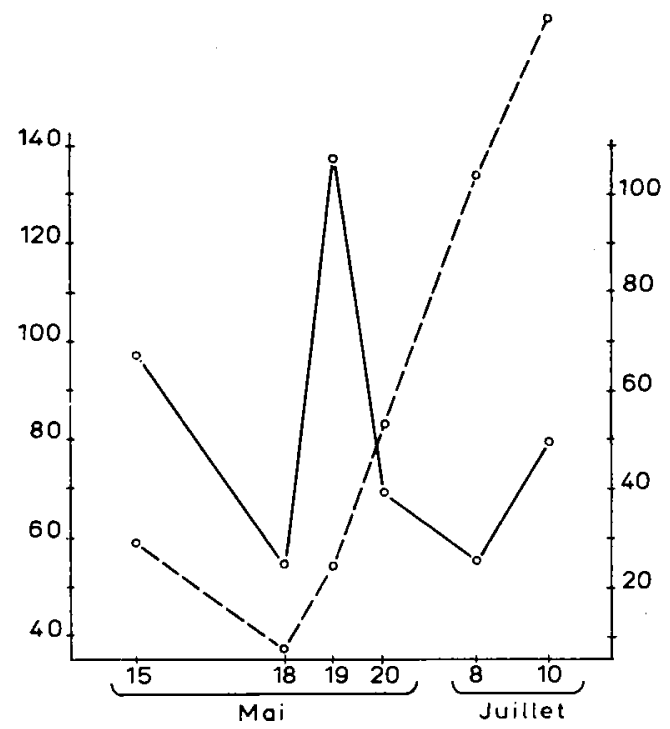

Fis. I2. - Recherche des rapports entre l'activité et la température; même méthode qu'en II, mais les graphiques s'inspirent ici des chiffres de LcNDIE. (En pointille, activité; en traits pleins, température).

trouvais pas en présence d'un artefact. J'ai donc repris les courbes de LundiE qui ne portent malheureusement que sur peu de jours, et je leur ai fait subir le même traitement d'intégration après calquage. On voit bien que, là non plus, il n'existe pas de corrélation entre les températures et les activités mesurées par une méthode tout à fait différente. LUNDIE signale lui-même que le degré de luminosité semble plus important que la température. J'ai donc porté sur la fig. I2 les chiffres relevés à l'actinomètre de BELLANi (car je ne disposais pas de solarigraphe). Mais, là non plus, on ne peut déceler aucune corrélation avec l'activité. LoUvEAuX (I958) remarque que l'activité de récolte du pollen n'a pas non plus de rapports directs avec la température. 


\section{L'ÉVAPORATION D'EAU}

La ruche évapore une quantité d'eau relativement importante, dont mon dispositif ne collectait évidemment qu'une fraction. Je me suis assuré d'abord que rien ne passe lorsqu'on interrompt, par une lame de métal, la communication entre la ruche et la boîte à glace. J'ai calculé ensuite, en dehors des périodes de miellée évidemment, l'émission d'eau, en quelque sorte "métabolique ", puisqu'il n'y avait pas de provisions liquides à évaporer dans la ruche : elle était de $4 \mathrm{cc}$. à $4,5 \mathrm{cc}$ par jour. Lorsqu'on donne du sirop de sucre à $70 \mathrm{p}$. IOO, l'augmentation de la quantité d'eau évaporée est de $6,5 \mathrm{cc}$ par litre. Mais on ne peut en tirer grand chose au point de vue de la quantité de nectar effectivement récolté, parce que la teneur en eau du nectar est très irrégulière (surtout chez Castanea où les nectaires sont largement exposés aux agents atmosphériques); elle dépend de l'heure, du jour, de l'hygrométrie, etc., si bien qu'on retrouve ici les incertitudes de la pesée des ruches. La pesée journalière, en effet, n'indique dans l'immédiat rien de précis quant à la quantité de matières sucrées effectivement récoltées; il faut attendre plusieurs jours, que les ouvrières aient évaporé le surplus d'eau du nectar, pour obtenir une mesure plus exacte. Mais justement, et en dehors des périodes de miellée, il semble que la mesure de "l'eau métabolique " pourrait fournir des renseignements intéressants. C'est une variable qui n'a jamais été mesurée jusqu'à présent. Je ne dispose évidemment pas d'assez de chiffres pour en faire la théorie complète; je me bornerai à signaler la possibilité d'une telle mesure.

\section{LA RÉCOLTE DU POLLEN}

Elle n'a pas été poursuivie assez longtemps pour que j'en parle en détail; nous n'avons guère appris de nouveau par rapport aux données traditionnelles exposées en détail dans la thèse de LouvEAux (I958). Là aussi, toutefois, la corrélation avec les courbes d'activité générale ne paraît pas très nette. Par contre, la corrélation est bonne, comme Louveaux l'a déjà signalé, avec la surface du couvain. Il semble que l'état interne de la ruche informe toutes les activités de l'abeille bien plus que les circonstances météorologiques (entre certaines limites, évidemment).

\section{CONCLUSION ET RÉSUMÉ}

On décrit un dispositif de mesure simultanée de la surface du couvain, de la récolte du pollen, de l'évaporation de l'eau et de l'activité générale.

La chaleur que dégagent les butineuses entrant ou sortant, est assez forte pour agir sur un évaporimètre ou un thermomètre enregistreur.

Activité et évaporation ne sont pas en corrélation étroite avec la température extérieure, pas plus que la récolte du pollen.

Il existe une activité nocturne de l'abeille, au cours de certaines nuits, sans qu'on puisse établir encore le facteur qui détermine les sorties. 


\section{SUMMARY}

\section{MIMULTANEOLS RECORDING EXPERIMENTS OF THE MAIN PHENOMENA IN THE LIFE OF THE HIVE}

A hive enclosed in a single glass frame which permitted a continuous observation of the bees, even during hibernation, was described. Exterior heat control by means of a resistance enabled the experimenter to act at will on the development of the hive.

A series of apparatus could be connected with the hive for the simultaneous recording of the activities of leaving the hive, pollen gathering and water elimination. This had revealed that at certain periods the bees made sorties during the night. The surface of the brood-comb and its fluctuations throughout the year were measured at the same time.

\section{RÉFÉRENCES BIBLIOGRAPHIQUES}

Chavvin R., i950. Méthode dobservation continue de la ponte de la Reine chez les abeilles. L'A picult., Sect. Sci., I 2 p.

Cinauvin R., 1952. Nouvelles techniques d'enregistrement de l'activite de la ruche. L'Apicul., Sect. Sci., 9-I4.

Chauvin R., 1955. Les facteurs de ponte chez la reine d Abeille. Ins. Soc., 3, 499-504.

Chacvin R., 1960. Les substances actives sur le comportement à l'intérieur de la ruche. Ann. Abeille, 3, I 85-97.

Chauviv R., 1962. Sur le noircissement des vieilles cires. Ann. Abeille, 5, 65-7.

Faberge Al., r943. An apparatus for recording the bees leaving and entering a hive. J. Scient. Instrum., 10, 28.

Golllot C., I954. Action de la fuméc sur Apis mellifica. Ann. Épiphyties., 3, 259-271.

Lovveaux J., 1957. Recherches sur la récolte du pollen par les abeilles. Thèse (Paris).

LundiE A. E., 1925. The flight activities of the honey bee. U.S. Dep. Agric. Bull. n ${ }^{0}$ I.328. 